\title{
Estudo TACQual: avaliação e melhoria da qualidade da requisição de TC da coluna lombar
}

Ana Miranda, Ana Raquel Marques, Ângela Neves, Helena Martins, Joana Tavares, Nuno Amândio, Raquel Barradas, Rita Lopes

\section{RESUMO}

Objetivos: Caracterizar os principais motivos para requisição de tomografia computorizada (TC) da coluna lombar na Unidade Local de Saúde (ULS), ao nível dos cuidados de saúde primários (CSP), e intervir de forma a melhorar a qualidade da sua prescrição.

Tipo de estudo: Quasi-experimental, pré pós-intervenção.

Local: Seis unidades funcionais (UF) da ULS.

População: Quarenta e nove médicos especialistas de medicina geral e familiar (MGF).

Métodos: Foram analisadas todas as requisições de TC da coluna lombar efetuadas entre 1 de outubro de 2014 e 31 de março de 2015 , relativamente ao número de requisições e informação clínica acompanhante, a qual permitiu a classificação de «aceitável» ou «não aceitável» com base na evidência bibliográfica e no protocolo de articulação entre o Agrupamento de Centros de Saúde (ACeS) e o serviço de ortopedia. Em setembro de 2015 foi realizada uma intervenção clínica com a apresentação dos resultados obtidos e a divulgação do protocolo. Globalmente foi acordado um aumento de $20 \%$ das requisições «aceitáveis». Foram analisados os pedidos de TC efetuados posteriormente - entre 1 de outubro de 2015 e 31 de março de 2016; a eficácia da intervenção traduzir-se-ia numa diminuição do número absoluto de requisições e num aumento da proporção daquelas classificadas como «aceitáveis».

Resultados: Após a intervenção verificou-se uma redução em 39,5\% de TC requisitadas (de 387 para 234 ) e um aumento estatisticamente significativo da proporção de requisições classificadas como «aceitáveis» (de 7,7\% para 15,2\%,p=0,0091). Contudo, apesar deste aumento, a proporção de requisições consideradas «não aceitáveis» manteve-se elevada (84,8\%).

Conclusão: A escassez/ininteligibilidade da informação clínica poderá ter levado à subestimação do número de requisições «aceitáveis». Apesar dos resultados favoráveis será necessária uma nova intervenção no sentido de uma redução mais expressiva e sustentada de requisições «não aceitáveis».

Palavras-chave: Dor lombar; Cuidados de saúde primários; Melhoria da qualidade; Tomografia computorizada.

\section{INTRODUÇÃO}

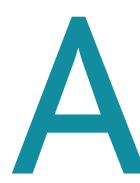

lombalgia aguda é um motivo de consulta frequente nos cuidados de saúde primários (CSP). ${ }^{1}$ Acomete cerca de 70 a $80 \%$ da população nalgum período da sua vida, constituindo uma das principais causas de dor crónica e de absentismo laboral. ${ }^{2-3} \mathrm{Em}$ cerca de $85 \%$ dos indivíduos não se identifica nenhuma patologia específica, pelo

Médicos Internos de Medicina Geral e Familiar. Unidade Local de Saúde de Matosinhos. que a lombalgia aguda, inespecífica ou com radiculopatia tem um curso tipicamente benigno e autolimitado, resolvendo-se com uma intervenção mínima. ${ }^{1,4-5} \mathrm{Na}$ avaliação inicial do doente com lombalgia aguda é essencial a investigação de sinais de alarme que podem indiciar uma patologia grave subjacente, nomeadamente síndroma da cauda equina, infeção, fratura, neoplasia (sobretudo metastática), espondilartropatia inflamatória e canal lombar estreito. ${ }^{6-11}$ A radiculopatia com duração superior a seis semanas, incapacitante e refratária ao tratamento médico, poderá requerer uma 
orientação especializada pela possibilidade de tratamento cirúrgico ou de realização de técnicas de radiologia de intervenção. ${ }^{6,12}$

A radiografia da coluna lombar é útil na avaliação de deformidade estrutural e, segundo algumas recomendações, constitui o exame inicial na lombalgia aguda com sinais de alarme e na lombalgia subaguda ou crónica. , $^{613-14}$ Porém, tem baixa sensibilidade e especificidade, ${ }^{1}$ sendo a ressonância magnética (RM) considerada o exame de eleição quando existem sinais de alarme $^{13-15}$ ou para confirmação de compressão radicular. ${ }^{8}$ Segundo a Norma n.o 047/2011, da Direção-Geral da Saúde, apesar de a TC constituir o exame de primeira linha ao nível dos CSP, ${ }^{16}$ alguns estudos sugerem a sua realização apenas em situações em que a RM esteja contraindicada ou inacessível pois, globalmente, a qualidade da informação acerca das estruturas a avaliar é inferior. ${ }^{1,14,17}$ Adicionalmente, não existem estudos que avaliem a acuidade da TC da coluna lombar na avaliação de patologia tumoral, infeciosa ou fraturas. ${ }^{18} \mathrm{O}$ efeito cumulativo da radiação ionizante, sobretudo em indivíduos mais jovens, é um fator que também não pode ser menosprezado. ${ }^{1,8,14,17-18}$

Segundo os investigadores e os profissionais do serviço de ortopedia do hospital de referência dos investigadores existe a perceção de uma prescrição inadequada de TC da coluna lombar nos CSP. Pensa-se que este exame seja frequentemente requisitado na ausência de sinais de alarme (ou face a uma evolução clínica favorável) e noutras situações em que, embora justifiquem a realização de exames de imagem, a sua orientação depende fundamentalmente do resultado da RM. Nestas situações, a realização da TC expõe os doentes a riscos desnecessários e acrescenta custos. Em 2014, no local de formação dos investigadores, houve um aumento em $67 \%$ do número de requisições de TC da coluna lombar com um custo associado de quase $53 \mathrm{mil}$ euros. De forma a prevenir as requisições inadequadas e a minimizar o seu impacto, em 2016 foi elaborado e aprovado um protocolo de referenciação para o serviço de ortopedia do hospital de referência, elaborado em conjunto pelos investigadores e pelos profissionais do serviço de ortopedia, definindo a adequação do uso de TC da coluna lombar e a orientação em cada situação particular de lombalgia, segundo as fontes bibliográficas supracitadas. Pensa-se que a difusão deste pro- tocolo entre os médicos de família (MF) e a sensibilização para as consequências do uso inadequado da TC da coluna lombar contribuirá para uma prescrição mais racional deste exame, salvaguardando a decisão do clínico em cada caso particular.

O objetivo deste trabalho consistiu na caracterização dos motivos subjacentes à requisição de TC da coluna lombar em seis unidades funcionais e a intervenção sobre a prática clínica, de forma a melhorar a qualidade da prescrição deste exame.

\section{MÉTODO}

Foi realizado um estudo quasi-experimental, com avaliação pré e pós-intervenção em seis unidades funcionais da ULS envolvendo um total de 49 médicos especialistas de MGF. Numa primeira fase foram analisadas todas as requisições de TC da coluna lombar efetuadas por estes profissionais durante seis meses (entre 1 de outubro de 2014 e 31 de março de 2015).

A maioria das TC solicitadas nas unidades funcionais foi realizada no hospital da ULS. A internalização destes exames permitiu a identificação pelo serviço de informática (através de códigos numéricos) daqueles efetuados durante o período de estudo. A partir do software SiiMA - Gestão de Serviços Clínicos, os autores acederam à versão digitalizada do documento anexo à requisição, que é preenchido manualmente pelo prescritor, contendo a informação clínica necessária para a realização do exame. Foi contabilizado o número absoluto de TC da coluna lombar requisitado em cada unidade funcional; foram recolhidos os dados referentes a variáveis demográficas (sexo e idade dos utentes), unidade funcional em que ocorreu a prescrição e informação clínica registada. Foram definidos os seguintes critérios de exclusão: 1) requisições cuja digitalização estava incompleta (ausência da segunda página contendo informação clínica); 2) ausência de preenchimento de qualquer informação clínica; 3) ilegibilidade da informação clínica; 4) requisições de TC da coluna lombar efetuadas por médicos internos de MGF (para assegurar que os médicos pré e pós-intervenção eram os mesmos, evitando enviesamento do número de prescrições); e 5) digitalização do pedido de exame referente a outro segmento da coluna vertebral.

Mediante a análise da informação clínica, as requisições de TC da coluna lombar foram classificadas pe- 
los autores em «Aceitável» ou «Não aceitável» (Quadro I), com base na evidência bibliográfica disponível e no protocolo supracitado. A informação clínica constante nos pedidos permitiria aplicar a categorização proposta no Quadro I. Contudo, tendo sido constatada a ausência frequente de informação clínica relativa à duração e evolução da sintomatologia nos casos de lombalgia e radiculopatia foram criadas as categorias «lombalgia sem outra especificação» e «radiculopatia sem outra especificação». Foi ainda adicionada a categoria «Outros» para classificar, por exemplo, as situações em que a TC foi solicitada para esclarecimento de alterações radiográficas sem clínica discriminada.

A colheita de dados e a classificação das requisições foi realizada por três dos investigadores, em separado. Sempre que existiam dúvidas na classificação a efetuar eram discutidas em conjunto, com base nos critérios de inclusão e exclusão.

Perante os resultados obtidos na primeira fase do estudo comprovou-se a necessidade de intervenção no sentido de melhorar a qualidade da prescrição da TC. Em setembro de 2015, os investigadores realizaram uma sessão clínica em cada uma das unidades funcionais participantes dirigida aos médicos, com cerca de meia hora de duração, que consistiu na apresentação dos resultados obtidos e numa breve revisão teórica acerca do uso apropriado da TC da coluna lombar, com divulgação do protocolo acima descrito e sensibilização para a necessidade de uma prescrição mais racional deste exame. Globalmente foi acordado entre os MF do ACeS um aumento de $20 \%$ das requisições «aceitáveis».

Numa segunda fase foram analisados os pedidos de TC efetuados após esta intervenção, entre 1 de outubro de 2015 e 31 de março de 2016, de modo semelhante ao descrito anteriormente e pelos mesmos três investigadores.

As variáveis foram codificadas, registadas e tratadas em suporte informático (Microsoft Excel® e IBM SPSS Statistics v. 23®). Através da aplicação do teste exato de Fisher foi feita uma análise comparativa dos resultados obtidos antes e após a intervenção. Considerou-se que a eficácia da intervenção traduzir-se-ia quer numa diminuição do número absoluto de requisições de TC da coluna lombar, quer num aumento da proporção daquelas requisitadas em conformidade com as reco- mendações clínicas, ambos estatisticamente significativos.

Foi obtido o parecer favorável da Comissão de Ética da ULS, bem como a autorização do Conselho de Administração para a realização do estudo, a 28 de julho de 2015. Durante todo o processo de recolha de dados foi garantida a confidencialidade dos participantes para elementos externos ao estudo. Os investigadores comprometeram-se à não utilização dos dados para outros fins. Em cada unidade funcional os resultados foram apresentados de forma global, sem particularização dos mesmos para cada médico. O estudo não implicou qualquer custo para a ULS.

\section{RESULTADOS}

Em ambos os períodos do estudo os utentes aos quais foi solicitada TC da coluna lombar eram maioritariamente do sexo feminino e tinham uma idade média próxima dos 50 anos (Quadro II) sendo que, nos períodos pré e pós-intervenção, 75 e $80 \%$ tinham idade inferior a 65 anos, respetivamente.

No período pré-intervenção foram requisitadas 387 TC da coluna lombar pelos 49 MF das seis unidades funcionais, das quais 38 foram excluídas da análise maioritariamente por digitalização incompleta ou por requisição efetuada por médicos internos. No período pós-intervenção foram requisitados 234 exames com exclusão de 30 da análise, principalmente pelos mesmos motivos referidos no período pré-intervenção (Quadros III e IV). A proporção de requisições excluídas foi semelhante nos dois períodos $(p=0,29)$. Globalmente verificou-se uma redução de 39,5\% ( $n=145)$ no número de TC requisitadas após a intervenção (Quadro III).

A classificação dos motivos de requisição de TC da coluna lombar nas seis unidades funcionais encontra-se no Quadro V. A radiculopatia, particularmente aquela «sem outra especificação», foi o motivo subjacente à maioria das requisições de TC da coluna lombar, representando aproximadamente $65 \%$ e $61 \%$ de todos os pedidos, no período pré e pós-intervenção, respetivamente. A lombalgia («sem outra especificação», crónica ou aguda inespecífica) configurou o segundo motivo mais frequente em ambos os períodos de estudo. Globalmente, a proporção de requisições aceitáveis duplicou após a intervenção, sendo a diferença estatisti- 


\section{QUADRO I. Indicações clínicas «aceitáveis» e «não aceitáveis» para a realização de TC da coluna lombar}

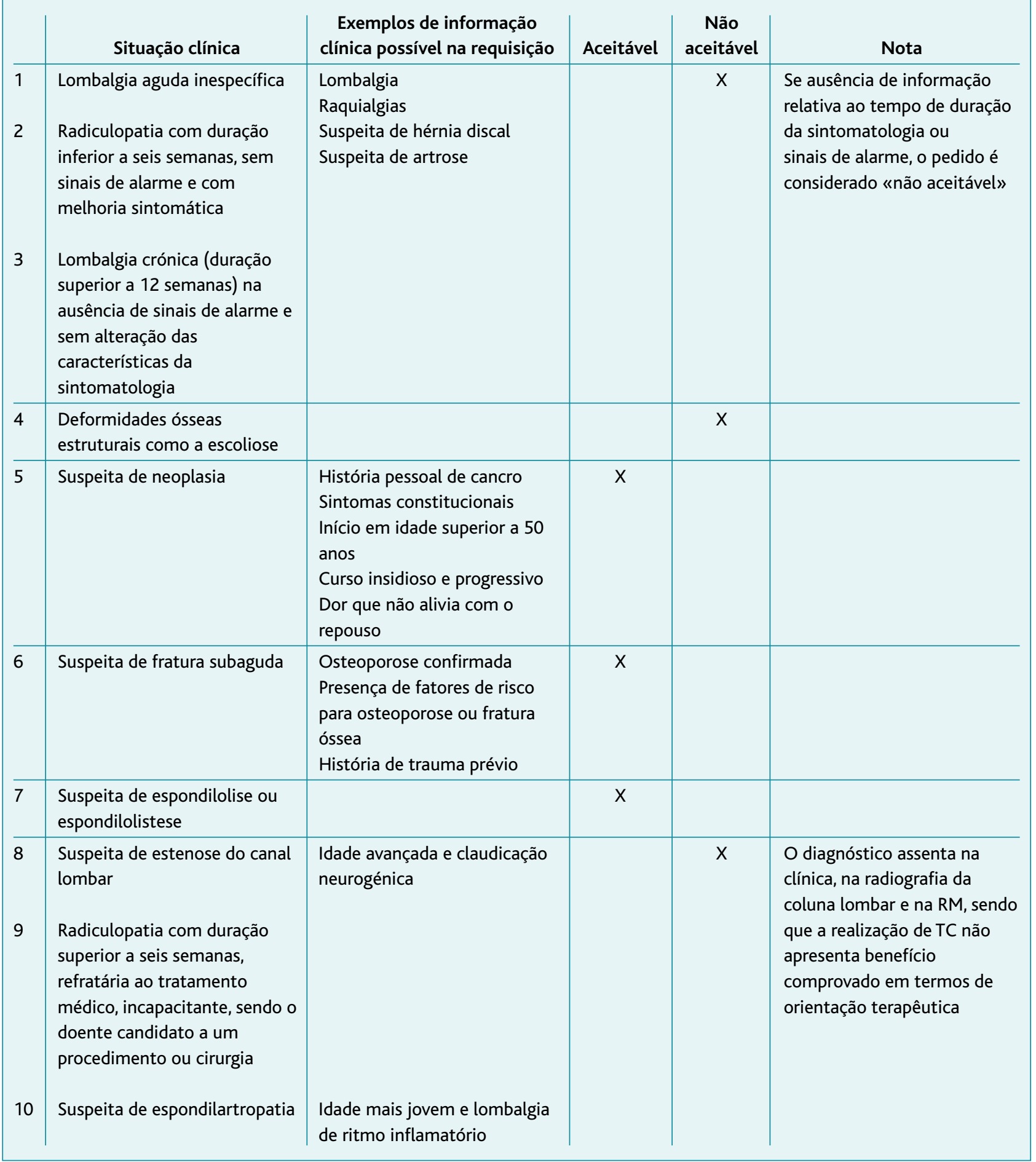




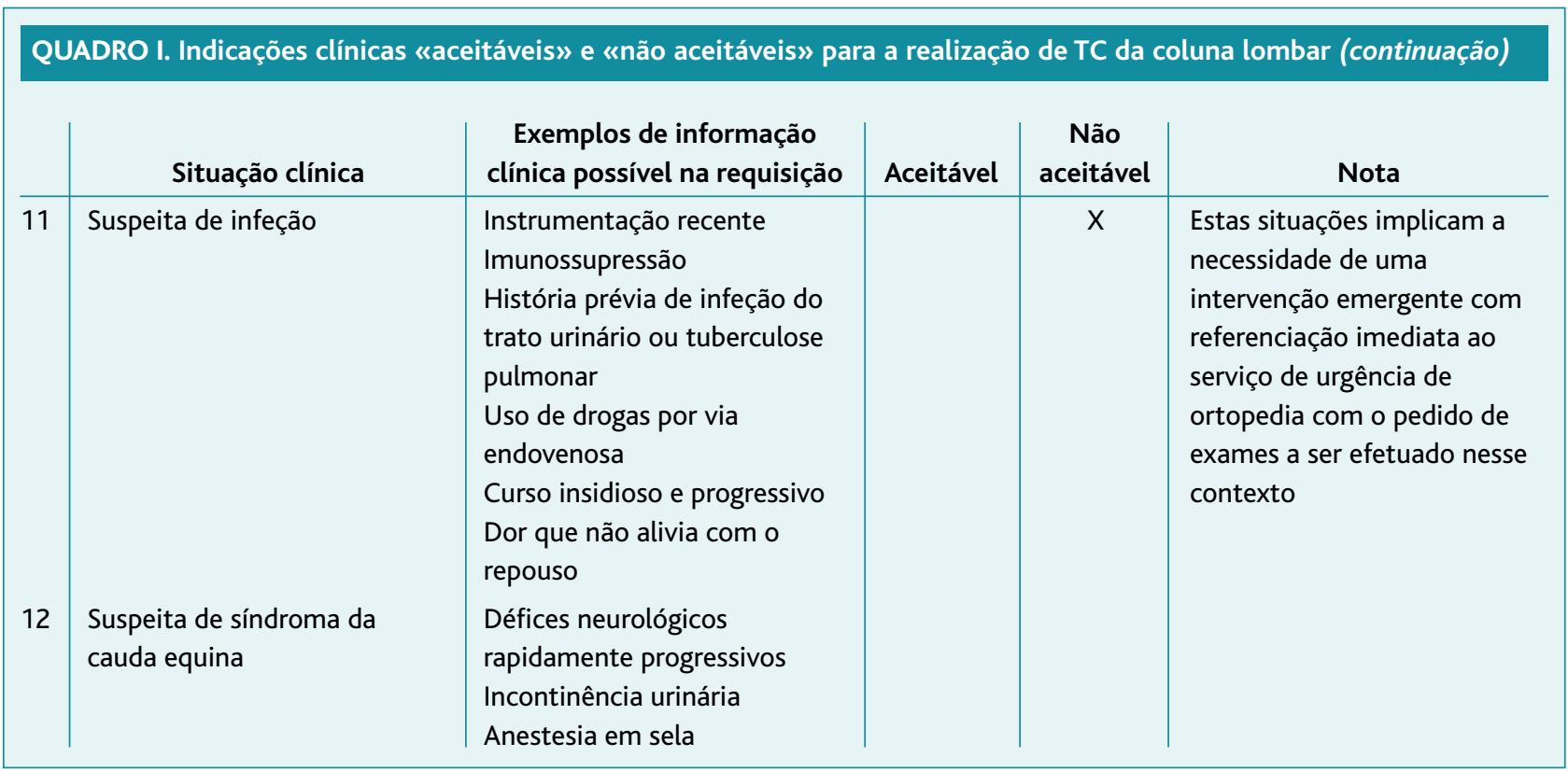

\begin{tabular}{|c|c|c|c|}
\hline & \multicolumn{2}{|c|}{ Sexo, $n(\%)$} & \multirow[t]{2}{*}{ Idade média (DP), anos } \\
\hline & Feminino & Masculino & \\
\hline Pré-intervenção & $214(61,3)$ & $135(38,7)$ & $52,1(14,6)$ \\
\hline Pós-intervenção & $139(68,5)$ & $64(31,5)$ & $53,1(13,8)$ \\
\hline
\end{tabular}

Nota: DP = desvio-padrão.

QUADRO III. Número total de requisições efetuadas e excluídas antes e após a
intervenção, por unidade funcional
\begin{tabular}{l|c|c|c|c|c|c|c} 
& UF 1 & UF 2 & UF 3 & UF 4 & UF 5 & UF 6 & Total \\
\hline Total requisições & & & & & & & \\
Pré-intervenção & 65 & 76 & 73 & 32 & 68 & 73 & 387 \\
Pós-intervenção & 29 & 50 & 41 & 12 & 42 & 60 & 234 \\
\hline Requisições excluídas & & & & & & & \\
Pré-intervenção & 6 & 9 & 6 & 8 & 3 & 6 & $38(9,8 \%)^{*}$ \\
Pós-intervenção & 3 & 10 & 4 & 3 & 5 & 5 & $30(12,8 \%)^{*}$ \\
\hline Requisições incluídas & & & & & & & \\
Pré-intervenção & 59 & 67 & 67 & 24 & 65 & 67 & 349 \\
Pós-intervenção & 26 & 40 & 37 & 9 & 37 & 55 & 204 \\
\end{tabular}

Nota: ${ }^{*} \mathrm{~N}^{\circ}$ de requisições excluídas $/ \mathrm{n}^{\circ}$ total de requisições efetuadas. camente significativa $(7,7 \%$ vs $15,2 \%, p=0,009)$. O QuadroVI apresenta, para cada unidade funcional, a proporção de requisições aceitáveis antes e após a intervenção.

\section{DISCUSSÃO}

Em ambas as fases do estudo, as situações clínicas que motivaram um maior número de requisições de TC da coluna lombar foram a radiculopatia e a lombalgia, sobretudo as categorias «sem outra especificação» - na ausência de informação sobre aspetos clínicos essenciais, nomeadamente sinais de alarme, assumiram-se como situações benignas nas quais o pedido de TC não era aceitável. Do mesmo modo, na ausência de informação adicional considerou-se que a realização de TC nas situações de radiculopatia ou lombalgia de evolução aguda ou crónica também não seria adequada. Não havendo evidência de um benefício claro, a prescrição de TC nestas situações resultou na exposição a radiação de uma população relativamente jovem (idade 


\begin{tabular}{|c|c|c|c|c|c|c|}
\hline $\begin{array}{l}\text { Motivos de } \\
\text { exclusão }\end{array}$ & $\begin{array}{l}\text { Digitalização } \\
\text { incompleta } n\end{array}$ & $\begin{array}{c}\text { Ausência de } \\
\text { informação } \\
\text { clínica } n\end{array}$ & $\begin{array}{c}\text { Ilegibilidade da } \\
\text { informação } \\
\text { clínica } n\end{array}$ & $\begin{array}{l}\text { Requisição por } \\
\text { médico interno } \\
n\end{array}$ & $\begin{array}{c}\text { Requisição relativa } \\
\text { a outro segmento } \\
\text { da coluna } n\end{array}$ & $\begin{array}{c}\text { Total } \\
n\end{array}$ \\
\hline Pré-intervenção & 12 & 2 & 1 & 19 & 4 & 38 \\
\hline Pós-intervenção & 12 & 2 & 4 & 10 & 2 & 30 \\
\hline
\end{tabular}

\section{QUADRO V. Classificação dos motivos de requisição de TC da coluna lombar nas seis unidades funcionais}

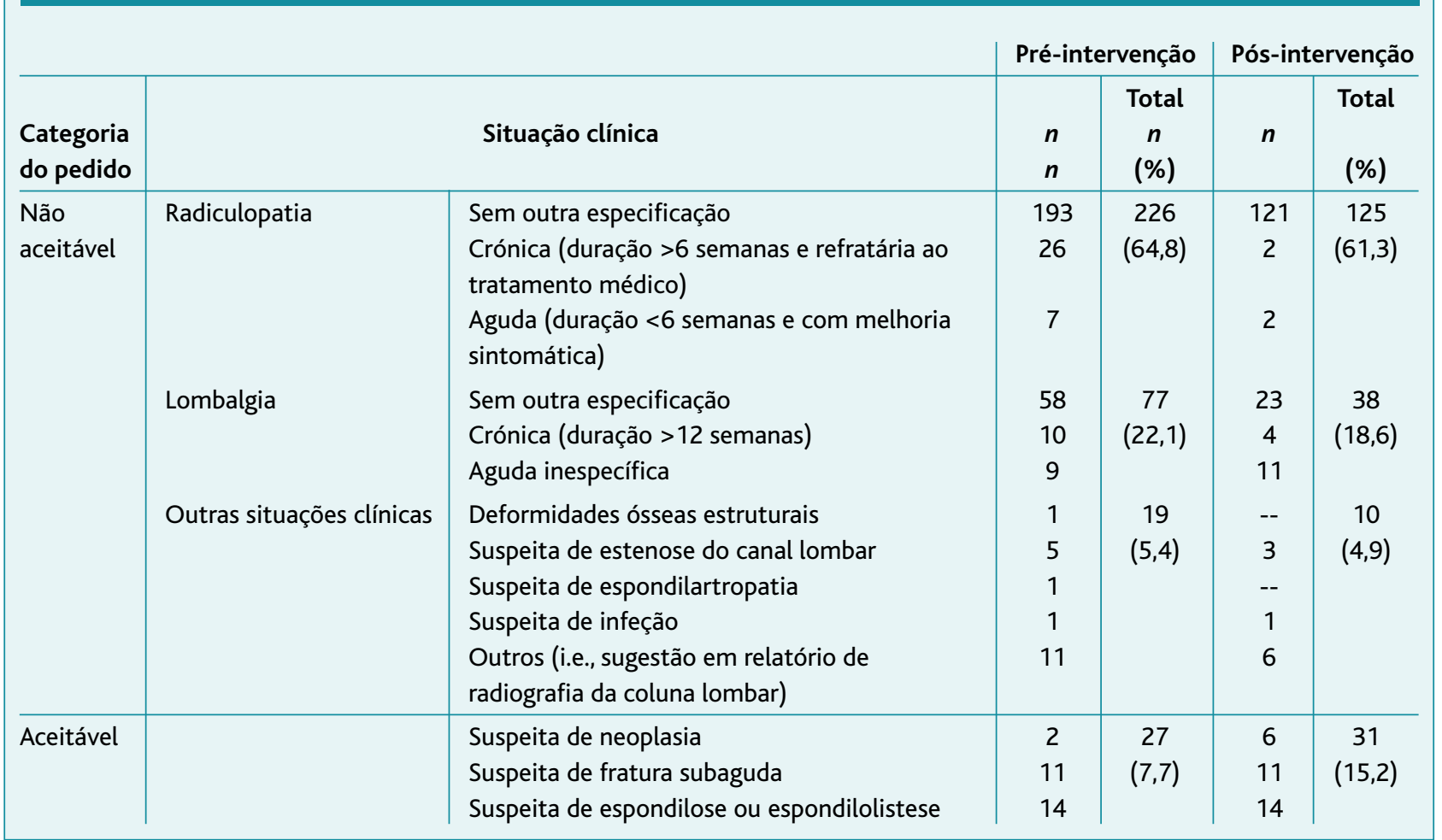

\begin{tabular}{|c|c|c|c|c|c|c|c|}
\hline & UF 1 & UF 2 & UF 3 & UF 4 & UF 5 & UF 6 & Total \\
\hline Pré-intervenção, $n$ (\%) & $3(5,1)$ & $8(11,9)$ & $5(7,5)$ & $1(4,2)$ & $8(12,3)$ & $2(2,9)$ & $27 / 349(7,7 \%) \dagger$ \\
\hline Pós-intervenção, $n$ (\%) & $2(7,7)$ & $7(17,5)$ & $5(13,5)$ & 0 (NA) & $15(40,5)$ & $2(3,6)$ & $31 / 204(15,2 \%) \dagger$ \\
\hline
\end{tabular}

Nota: † Número de requisições da categoria «aceitável»/ número de requisições incluídas.

média de cerca de 50 anos) e na qual é também elevada a probabilidade de achados imagiológicos que não se correlacionam com a sintomatologia nem alteram a sua história natural.
Na ausência de uma educação adequada acerca da prevalência, tratamento e prognóstico, o indivíduo poderá fixar-se apenas nestes achados imagiológicos ao invés de adotar um papel ativo no seu plano terapêuti- 
co, aumentando o risco de progressão para cronicidade. ${ }^{6}$ Contudo, é de salientar que a ausência ou clareza insuficiente da informação clínica poderá ter levado à sobrestimação do número de requisições classificadas como «não aceitáveis». Adicionalmente, ao ter sido realizada por três dos investigadores, poderá existir alguma subjetividade subjacente à própria classificação em «aceitável» ou «não aceitável». Para minimizar este viés, as situações dúbias foram discutidas em conjunto tendo sido possível efetuar uma classificação final em unanimidade.

Após a intervenção houve uma redução do número de exames solicitados em 39,5\% e o número de requisições consideradas «aceitáveis» aumentou significativamente (duplicou), cumprindo-se assim o objetivo previamente estabelecido. Pela associação temporal, os resultados deveram-se provavelmente à intervenção efetuada, embora não seja possível estabelecer uma associação causal definitiva dada a inexistência de um grupo de controlo não exposto à intervenção. Apesar dos resultados favoráveis, a proporção de requisições classificadas como «não aceitáveis» manteve-se elevada tanto antes $(92,3 \%)$ como após a intervenção $(84,8 \%)$. Decorre daqui a necessidade de perceber os motivos subjacentes à persistência da requisição de TC nestas situações. Hipotetizam-se fatores relacionados quer com o utente, quer com o clínico. O primeiro, pelo impacto na qualidade de vida e/ou receio de um problema grave, poderá depositar as suas expectativas na imagiologia, sobretudo na mais avançada. Embora pouco frequentes existem causas potencialmente graves de lombalgia, mas que poderão ser descortinadas através de uma história clínica minuciosa. Caso o médico não se encontre na posse de todas as competências clínicas necessárias para explorar este sintoma, poderá encontrar na imagiologia um subterfúgio. Consequentemente poderá ser necessário o incremento da formação dos MGF nesta área. Frequentemente, a realização de TC da coluna lombar é sugerida pelo médico radiologista (i.e., nos relatórios de radiografia da coluna lombar), consoante o contexto clínico, o que sugere uma necessidade de comunicação e articulação com outras especialidades.

A metodologia deste trabalho foi desenvolvida num contexto específico, em articulação com o serviço de ortopedia do hospital de referência, que se traduziu na elaboração de um protocolo de referenciação que poderá não ser aplicável noutras realidades. Contudo, este estudo fomentou a discussão entre dois níveis de cuidados acerca de um problema frequente, permitindo a identificação de situações nas quais é possível uma prescrição mais racional de TC da coluna lombar pelos MF.

\section{AGRADECIMENTOS}

Ao Dr. Nuno Alves, presidente do Conselho Clínico do ACeS, pela disponibilização dos dados informáticos e articulação com o serviço de ortopedia. Ao Dr. Diogo Libânio pela revisão final do trabalho.

\section{REFERÊNCIAS BIBLIOGRÁFICAS}

1. Casazza BA. Diagnosis and treatment of acute low back pain. Am Fam Physician. 2012;85(4):343-50.

2. Airaksinen O, Brox Jl, Cedraschi C, Hildebrandt J, Klaber-Moffett J, Kovacs $F$, et al. European guidelines for the management of chronic nonspecific low back pain. Eur Spine J. 2006;15 Suppl 2:s192-300.

3. Froud R, Patterson S, Eldridge S, Seale C, Pincus T, Rajendran D, et al. A systematic review and meta-synthesis of the impact of low back pain on people's lives. BMC Musculoskelet Disord. 2014;15:50.

4. Henschke N, Maher CG, Ostelo RW, de Vet HC, Macaskill P, Irwig L. Red flags to screen for malignancy in patients with low-back pain. Cochrane Database Syst Rev. 2013;(2):CD008686.

5. Williams CM, Henschke N, Maher CG, van Tulder MW, Koes BW, Macaskill $P$, et al. Red flags to screen for vertebral fracture in patients presenting with low-back pain. Cochrane Database Syst Rev. 2013;(1): CD008643.

6. Low back disorders. In: Hegmann KT, editor. Occupational medicine practice guidelines: evaluation and management of common health problems and functional recovery in workers. 3rd ed. Elk Grove Village, IL: American College of Occupational and Environmental Medicine; 2011. ISBN 9780615452272

7. Michigan Quality Improvement Consortium. Michigan quality improvement consortium guideline: management of acute low back pain [Internet]. Southfield (MI): MQIC; 2012.Available from: http://mqic.org/ pdf/mqic_management_of_acute_low_back_pain_cpg.pdf

8. Davis PC, Wippold FJ 2nd, Brunberg JA, Cornelius RS, De La Paz RL, Dormont PD, et al. ACR appropriateness criteria on low back pain. J Am Coll Radiol. 2009;6(6):401-7.

9. Goertz M, Thorson D, Bonsell J, Bonte B, Campbell R, Haake B, et al. Adult acute and subacute low back pain. 15th ed. Bloomington, MN: Institute for Clinical Systems Improvement; 2012.

10. Institute of Health Economics. Guideline for the evidence-informed primary care management of low back pain [Internet]. Edmonton (AB): IHE; 2009. Available from: http://nationalpaincentre.mcmaster.ca/documents/LowBackPainGuideline.pdf

11. Chiodo AE, Alvarez DJ, Graziano GP, Haig AJ, Van Harrison R, Park P, et al. Acute low back pain [Internet]. Ann Arbor, MI: Michigan Medicine, University of Michigan; 2010. Available from: http://www.med.umich. edu/1info/FHP/practiceguides/back/back.pdf 
12. Kreiner $\mathrm{S}$, editor. Clinical guidelines for diagnosis and treatment of lumbar disc herniation with radiculopathy [Internet]. Burr Ridge, IL: North American Spine Society; 2012. Available from: https://www.spine .org/Documents/ResearchClinicalCare/Guidelines/LumbarDiscHerniation.pdf

13. Koes BW, van Tulder M, Lin CW, Macedo LG, McAuley J, Maher C. An updated overview of clinical guidelines for the management of nonspecific low back pain in primary care. Eur Spine J. 2010;19(12):207594.

14. Last AR, Hulbert K. Chronic low back pain: evaluation and management. Am Fam Physician. 2009;79(12):1067-74.

15. Savigny $P$, Watson $P$, Underwood $M$. Early management of persistent non-specific low back pain: summary of NICE guidance. BMJ. 2009;338:b1805.

16. Direção-Geral da Saúde. Imagiologia da coluna vertebral: tomografia computorizada da coluna - Norma da Direção-Geral da Saúde n. ${ }^{\circ}$ 047/2011, de 26/12/2011. Lisboa: DGS; 2011.

17. Chou R, Qaseem A, Snow V, Casey D, Cross JT Jr, Shekelle P, et al. Diagnosis and treatment of low back pain: a joint clinical practice guideline from the American College of Physicians and the American Pain Society. Ann Intern Med. 2007;147(7):478-91.

18. Van Rijn RM, Wassenaar M, Verhagen AP, Ostelo RW, Ginai AZ, de Boer
$M R$, et al. Computed tomography for the diagnosis of lumbar spinal pathology in adult patients with low back pain or sciatica: a diagnostic systematic review. Eur Spine J. 2012;21(2):228-39.

\section{CONFLITO DE INTERESSES}

Os autores declaram não ter quaisquer conflitos de interesse.

\section{FINANCIAMENTO}

O estudo foi desenvolvido pela Unidade de Epidemiologia do Instituto de Medicina Preventiva e Saúde Pública, da Faculdade de Medicina de Lisboa (IMPSP-FML), e cofinanciado pela Fundação para a Ciência e Tecnologia (FCT), através do projeto PTDC/SAU-ESA/103511/2008, e pela Fundação AstraZeneca. O trabalho de Marília Antunes é parcialmente financiado pela FCT, através do projeto UID/MAT/00006/2013.

\section{ENDEREÇO PARA CORRESPONDÊNCIA \\ Ana Miranda \\ E-mail: anarpmiranda@hotmail.com \\ http://orcid.org/0000-0002-7271-5238}

Recebido em 01-02-2017

Aceite para publicação em 27-03-2018

\section{ABSTRACT}

\section{TACQUAL STUDY: ASSESSMENT AND IMPROVEMENT OF THE REQUESTS OF LUMBAR SPINE CT SCANS}

Aim: To characterize the main reasons for requesting lumbar spine computed tomography (CT) scans at primary care units within a Local Health Unit (ULS), and to intervene in order to improve the quality of such requests.

Type of study: Quasi-experimental, pre- post assessment.

Local: Six Functional Units (UF) of ULS.

Population: Forty-nine family physicians.

Methods: All the lumbar spine CT scans performed between October 1, 2014 and March 31, 2015 were reviewed regarding the number of requests and clinical information, which allowed their classification as "acceptable" or "not acceptable", accordingly to the current scientific evidence, and to the protocol between ACeS and orthopedics department of ULS. In September 2015, a clinical education session was carried out at each of the participating units, where the results were presented and the protocol disseminated. A $20 \%$ increase in the number of 'acceptable' request was agreed. A second assessment was performed evaluating the CT scans requested by the same family physicians between October 1, 2015 and March 31, 2016. Efficacy of the intervention was defined as a decrease in the absolute number of CT scan requests, and the increase of the proportion of 'acceptable' requests.

Results: After the intervention, a 39.5\% reduction in the overall number of CT scans requested was observed (387 vs 234 ), and a statistically significant increase in the number of requests classified as 'acceptable' $(7.7 \%$ vs $15.2 \%, p=0.0091)$. However, despite this increase, the proportion of requests classified as 'not acceptable' remained high (84.8\%).

Conclusion: The scarcity/unintelligibility of clinical information may have led to the underestimation of the number of 'acceptable' requests. In spite of the favorable outcomes, a new intervention will be necessary for a more significant and sustained reduction of the proportion of 'not unacceptable' requests.

Keywords: Low back pain; Primary care; Improve of quality; Computed tomography scan. 